\title{
On the Relation between Message Sentiment and its Virality on Social Media
}

\author{
Sho Tsugawa · Hiroyuki Ohsaki
}

Received: date / Accepted: date

\begin{abstract}
We investigate the relation between the sentiment of a message on social media and its virality, defined as the volume and speed of message diffusion. We analyze 4.1 million messages (tweets) obtained from Twitter. Although factors affecting message diffusion on social media have been studied previously, we focus on message sentiment and reveal how the polarity of message sentiment affects its virality. The virality of a message is characterized by the number of message repostings (retweets) and the time elapsed from the original posting of a message to its $N$ th reposting ( $N$-retweet time). Through extensive analysis using the 4.1 million tweets and their retweets in one week, we discover that negative messages are likely to be reposted more rapidly and frequently than positive and neutral messages. Specifically, the reposting volume of negative messages is $20-60 \%$ higher than that of positive and neutral messages, and negative messages spread $25 \%$ faster than positive and neutral messages when the diffusion volume is quite high. We also perform longitudinal analysis of message diffusion observed over one year, and find that recurrent diffusion of negative messages is less frequent than that of positive and neutral messages. Moreover, we present a simple message diffusion model that can reproduce the characteristics of message diffusion observed in this paper.
\end{abstract}

Keywords Social media - Twitter - Information diffusion · Retweet $\cdot$ Sentiment

S. Tsugawa $\square$

Faculty of Engineering, Information and Systems, University of Tsukuba

1-1-1 Tennodai, Tsukuba, Ibaraki 305-8573, Japan

Tel./Fax: +81-29-853-5597

E-mail: s-tugawa@cs.tsukuba.ac.jp

H. Ohsaki

School of Science and Technology, Kwansei Gakuin University

\section{Introduction}

On social media, such as Twitter and Facebook, users post many messages including their opinions and feelings. One of the most successful social media services, Twitter, allows users to post tweets, which are short messages with a limit of 140 characters. As of early 2014, 240 million users were posting over 500 million tweets on Twitter each day (Yang et al., 2014).

Some of the messages posted on social media are disseminated to many other users by word-of-mouth, which affects trends and public opinions in society. Social media users can disseminate messages to their friends via functionalities, such as retweeting in Twitter and share in Facebook. This word-of-mouth message diffusion on social media is an important mechanism that influences public opinion and can affect brand awareness and product market share (Bakshy et al., 2011). Therefore, information diffusion in social media has attracted the attention of many researchers (Suh et al., 2010; Hong et al., 2011b; Naveed et al., 2011; Stieglitz and Dang-Xuan, 2012; Gruzd et al., 2011; Stieglitz and Dang-Xuan, 2013; Bakshy et al., 2012; Kempe et al., 2003).

As we will discuss in Section 2, factors affecting wordof-mouth message diffusion in social media have been analyzed extensively (Suh et al., 2010; Hong et al., 2011b; Naveed et al., 2011). Researchers often focus on Twitter as one of the largest social media services, and investigate the relation between features extracted from a tweet and its virality. For instance, it has been shown that tweets with features such as URLs, hashtags, and emotional words are more likely to be retweeted than those without these features (Naveed et al., 2011). It has also been shown that the tweet topic and the number of followers of the tweet publisher are major factors affecting tweet diffusion (Suh et al., 2010; Hong et al., 2011b). 
We focus on sentiment as a factor affecting message diffusion, and examine the effects of positive and negative sentiment in each tweet on its virality on Twitter. Behaviors of social media users are not necessarily objective and legitimate, and psychological and emotional factors are expected to affect the users' behaviors.

The relation between message sentiment and virality of a message, defined as the volume and the speed of the message diffusion, has been studied (Stieglitz and Dang-Xuan, 2012; Gruzd et al., 2011; Stieglitz and Dang-Xuan, 2013). However, different results have been reported for the volume of message diffusion. For instance, Gruzd et al. (2011) and Ferrara and Yang (2015) have shown that positive tweets are retweeted more than negative tweets, whereas Stieglitz and Dang-Xuan (2013) have shown the opposite. Moreover, most studies have focused on only the volume of diffusion and not on the diffusion speed. Although Stieglitz and DangXuan (2013) and Ferrara and Yang (2015) have performed pioneering work in analyzing the relation between tweet sentiment and diffusion speed, their analyses used the time interval between the original tweet and only the first retweet as a measure of diffusion speed.

This paper aims to reveal how the sentiment of a tweet affects its virality in terms of both diffusion volume and speed on Twitter by using a large-scale dataset containing 4.1 million tweets. Our main contributions are as follows.

- Through analysis of 4.1 million non-domain-specific tweets, we reveal that negative messages are typically more viral, in terms of both diffusion volume and speed, than positive and neutral messages. Psychology studies suggest that negative things have a stronger effect on people than positive things (Taylor, 1991; Baumeister and Bratslavsky, 2001; Rozin and Royzman, 2001). We provide empirical evidence for the existence of such bias on social media. In particular, to the best of our knowledge, this is the first study to investigate the relation between the sentiment and diffusion speed of tweets with a high diffusion volume.

- We also reveal that, as a result of their high virality, negative messages typically spread with a single burst, whereas some positive and neutral messages spread recurrently with multiple bursts. Through a longitudinal analysis of tweet diffusion observed over one year, we discover that some tweets spread recurrently with multiple bursts, although most tweets spread with only a single burst. We show that the ratio of tweets with multiple bursts for negative tweets is significantly lower than those for positive and neutral tweets.

- We present a simple model of message diffusion that can reproduce the characteristics observed in this paper. We extend a popular influence cascade model, the independent cascade (IC) model, to include time delays for message diffusion. The model can reproduce the dif- ferences of diffusion volume and speed among different sentiments by adjustment of parameters.

This paper is an extended version of our previous conference paper (Tsugawa and Ohsaki, 2015). We have extended the observation period of retweets from one week to one year, performed extensive analyses (Section 4.3), and constructed a message diffusion model that can reproduce the characteristics of message diffusion observed in this paper (Section 5).

The remainder of the paper is organized as follows. Section 2 introduces works related to analyses of message diffusion on social media. Section 3 explains the methodology and dataset used for the analyses. Section 4 shows the results, and Section 5 presents a model that can reproduce our results. Section 6 discusses the implications of the results and limitations of the work. Finally, Section 7 contains our conclusions.

\section{Related Work}

Factors affecting retweetability of tweets (i.e., probability of retweet) have been analyzed in previous works (Suh et al., 2010; Naveed et al., 2011; Hansen et al., 2011). Suh et al. (2010) analyzed 74 million tweets, and showed that the presence of hashtags and URLs significantly affects retweetability, whereas the number of past tweets does not. Naveed et al. (2011) analyzed 60 million tweets, and showed that the presence of emotional words, hashtags, and URLs are major factors affecting retweetability.

Hansen et al. (2011) investigated the relation between emotions contained in a tweet and retweetability. Analysis of approximately 560,000 tweets showed that for tweets about news, negative tweets have higher retweetability than positive tweets, whereas the opposite is true for non-newsrelated tweets. These studies have focused on retweetability; however, in our work, we have focused on the volume and speed of retweets.

Factors affecting the volume of retweets have been analyzed (Hong et al., 2011b; Gruzd et al., 2011; Stieglitz and Dang-Xuan, 2013). Hong et al. (2011b) have shown that tweet topics determined by topic modeling, which is a widely used natural language processing technique (Blei et al., 2003), and the number of followers of the tweet publisher are useful features for predicting the volume of retweets.

The relation between tweet sentiment and the volume of tweet diffusion has been examined (Gruzd et al., 2011; Stieglitz and Dang-Xuan, 2013, 2012). Gruzd et al. (2011) have analyzed 46,000 tweets related to the Winter Olympics in 2010, and discovered that positive tweets had a higher number of retweets than negative tweets. In contrast, 
Stieglitz and Dang-Xuan (2013, 2012) have analyzed approximately 170,000 tweets related to political elections in Germany, and revealed that negative and positive tweets had a higher volume of retweets than neutral tweets. Moreover, in one dataset, they showed that negative tweets had a higher volume of retweets than positive tweets, whereas in the other, there was no significant difference in retweet volume between positive and negative tweets (Stieglitz and DangXuan, 2013). These studies used domain-specific tweets, where the tweets were related to specific social events, and reached different conclusions. Ferrara and Yang (2015) have analyzed non-domain-specific tweets of English-speaking users, and shown that positive tweets had a higher volume of retweets than negative tweets. Similarly to Ferrara and Yang (2015), our study uses larger-scale non-domainspecific tweets of Japanese-speaking users, and investigates the general relation between the sentiment of a tweet and diffusion volume.

Analyses of the relation between message sentiment and diffusion speed are limited. Stieglitz and Dang-Xuan (2013) and Ferrara and Yang (2015) have investigated the relation between tweet sentiment and retweet speed. They used the time interval between the original tweet and the first retweet (1-retweet time) as a measure of retweet speed, and Stieglitz and Dang-Xuan (2013) found no significant difference between the retweet speeds of positive and negative tweets, whereas Ferrara and Yang (2015) found that the retweet speed was faster in negative tweets than in positive tweets. Extending the methodology of their work, we used the time interval between the original tweet and the $N$ th retweet as a measure of diffusion speed, and investigated the effects of message sentiments on diffusion speed.

Research into predicting the volume of retweets is related and is an active topic (Cheng et al., 2014; Kupavskii et al., 2012). Cheng et al. (2014) predicted the volume of retweets by applying machine-learning techniques. Although these studies have constructed prediction models that use several features, we examine the effect of the feature (message sentiment in this study) on retweet volume. Our results can be used to predict the retweet volume and provide several suggestions for improving marketing and designing new functionality in social media, as discussed in Section 6.

\section{Methodology}

\subsection{Overview}

We collected tweets on Twitter, and investigated the relation between the sentiment of each tweet and its virality. To focus on users with the same culture and to eliminate the effects of different time-zones, we restricted our research to tweets from Japanese twitter users. Following the method in (Stieglitz and Dang-Xuan, 2013), we categorized the tweet sentiment as positive, negative, or neutral.

Tweet sentiment was determined by use of two methods: automated classification using a dictionary of positive and negative words (Takamura et al., 2006, 2005), and manual classification by several people. For automated classification, we determined the sentiment of each tweet by counting the number of affective words used in the tweet. Since such automated classification could cause classification errors, we also used manual classification of a subset of collected tweets. The two classification methods were used to check the robustness of the results. Details of these methods are explained in Section 3.3.

For each original tweet, we calculated the number of retweets and the time interval between the original tweet and the $N$ th retweet ( $N$-retweet time). We investigated the relation between these measures and tweet sentiment, which will be introduced in Sections 4.1 and 4.2. Moreover, we also investigated the relation between the sentiment of a tweet and the temporal pattern of its longitudinal diffusion, which will be introduced in Section 4.3.

\subsection{Dataset}

Using the Twitter application programming interface (API), we collected Japanese retweets posted during July 25-31 $2013^{1}$. Retweets where the original tweet was posted before July 25, 2013 were discarded. For each original tweet, we counted the number of retweets and extracted original tweets that were retweeted multiple times; namely, tweets with a retweet number of greater than one. This was intended to focus on tweets with a certain amount of retweet volume. We obtained 4,285,037 original tweets, referred to hereafter as tweets. There were no special social events such as the Olympic or political elections during the period of data collection. The distribution of the number of retweets in the dataset is shown in Table 1. Table 1 shows that our dataset included tweets with a high diffusion volume. Because the distribution of the number of retweets for a tweet is heavy-tailed (Kwak et al., 2010) and an occurrence of a high degree of retweet diffusion is a rare event, previous studies (Gruzd et al., 2011; Stieglitz and Dang-Xuan, 2013) have used tweets with relatively small diffusion. In contrast, by collecting a large number of tweets, our dataset has included a sufficient number of tweets with a high diffusion volume, allowing us to analyze $N$-retweet time for a large retweet count, $N$.

From the 4,285,037 tweets, we chose 8,000 tweets for determining sentiment by manual evaluations. To obtain the 8,000 tweets, we used stratified sampling, instead of pure

1 We used the Search API in Twitter REST API v1.1, and collected Japanese tweets using the query $q=R T$, lang=ja. 
Table 1 Distribution of number of retweets in dataset

\begin{tabular}{r|r|r}
\hline Section & Number of retweets & Number of tweets \\
\hline 1 & $2-10$ & $3,748,449$ \\
2 & $11-25$ & 318,640 \\
3 & $26-50$ & 111,527 \\
4 & $51-75$ & 37,174 \\
5 & $76-100$ & 18,616 \\
6 & $101-250$ & 33,847 \\
7 & $251-500$ & 10,359 \\
8 & $501-750$ & 2,903 \\
9 & $751-1000$ & 1,227 \\
10 & 1001 or more & 2,295 \\
\hline
\end{tabular}

Table 2 Statistics for collected tweet dataset, $D_{A}$

\begin{tabular}{c|rrr}
\hline \hline & Mean & Median & Std. dev. \\
\hline Number of retweets & 9.70 & 3 & 70.80 \\
Number of URLs & 0.39 & 0 & 0.53 \\
Number of hashtags & 0.27 & 0 & 0.70 \\
Number of followers & 6237.30 & 515 & 36220.61 \\
\hline
\end{tabular}

random sampling, to extract tweets with different diffusion volumes. We classified all tweets into 10 sections, as shown in Table 1, and randomly chose 800 tweets for each section. We denote the dataset of all tweets and their retweets during July 25-31, 2013, as $D_{A}$, and the 8,000 sampled tweets and their retweets during July 25-31, 2013, as $D_{S}$. We used dataset $D_{A}$ and $D_{S}$ for the analysis of the relation between tweet sentiment and measures of tweet virality in Sections 4.1 and 4.2. Statistics about the collected tweet data, $D_{A}$, are shown in Table 2 .

We also collected additional data for analyzing the relation between tweet sentiment and temporal pattern of longitudinal tweet diffusion. We collected retweets of the 4,285,037 tweets posted during one year (i.e., from August 1, 2013, to July 31, 2014). We then obtained 3,727,231 retweets (i.e., 0.87 retweets per tweet). We denote the dataset of all tweets and their retweets between July 25, 2013, and July 31,2014 , as $D_{A L}$, and 8,000 sampled tweets and their retweets between July 25, 2013, and July 31, 2014, as $D_{S L}$. Datasets $D_{A L}$ and $D_{S L}$ were used for the analyses in Section 4.3.

\subsection{Methods for Inferring Tweet Sentiment}

We inferred the sentiment of each tweet in dataset $D_{A}$ by using a dictionary of affective words. The dictionary is compiled by manual evaluation of a dictionary of positive and negative words extracted according to a technique in (Takamura et al., 2006, 2005). The dictionary contains 2,871 positive words and 3,534 negative words. Examples of words are show in Tab. 3. We used MeCab ${ }^{2}$ for morphological

\footnotetext{
2 http://taku910.github.io/mecab/
}

Table 3 Examples of positive and negative words. English translations for dictionary-listed Japanese words are shown.

\begin{tabular}{|c|c|}
\hline Positive & Negative \\
\hline $\begin{array}{l}\text { Happy, laugh, pretty, favorite } \\
\text { good, comfortable, smile } \\
\text { celebrate, beautiful, love }\end{array}$ & $\begin{array}{l}\text { Sad, dislike, sick, fear } \\
\text { bad, horrible, tired } \\
\text { unlucky, anxiety, sorry }\end{array}$ \\
\hline
\end{tabular}

stemming of the Japanese tweet text, and obtained words used in each tweet. For each tweet, we counted the number of positive and negative words listed in the dictionary. We classified each tweet according to the following rules: A tweet that had at least one positive word and no negative words was positive; a tweet that had at least one negative word and no positive words was negative; a tweet that had no positive or negative words was neutral; other tweets were discarded. Following these rules, we obtained 863,830 positive tweets, 343,910 negative tweets, 2,929,324 neutral tweets, and 147,973 tweets that were discarded. Previous studies (Naveed et al., 2011; Hansen et al., 2011) have used similar dictionary-based approaches to analyze the relation between tweet sentiment and virality. Therefore, this approach is reasonable for classifying large-scale tweet data.

Moreover, we inferred the sentiment of each tweet in dataset $D_{S}$ by manual evaluation. We recruited 11 annotators from undergraduate and graduate students in our laboratory. Annotators were instructed to read the tweets independently, and to tag each tweet as positive, negative, neutral, or uncertain. For each tweet, three annotators independently gave a sentiment tag for the tweet. Following the method used in the sentiment analysis task in the SemEval workshop (Rosenthal et al., 2015), we adopted a majority vote for determining the sentiment label for each tweet. We discarded tweets that were given three different tags by the three annotators, and tweets that were given two or more uncertain tags. If two of the three annotators gave the tweet the same tag, the tweet was classified as having the sentiment corresponding to the tag. Using this method, we obtained 1,436 positive tweets, 976 negative tweets, and 4,734 neutral tweets (for a total of 7,146 tweets), which were used in the analyses. We discarded 854 tweets, of which 138 tweets were uncertain.

We examined the agreement between the automated classification using the dictionary of affective words, and the manual classification (Table 4). The overall agreement between the automated and manual classifications was approximately $60 \%$. Evaluating the sentiment of short messages automatically is challenging (Barbosa and Feng, 2010), and overall agreement is often low. However, the proportion of tweets classified as being of the opposite sentiment was only $2 \%$, suggesting that automated classification can be used for our analysis, particularly for comparing negative and positive tweets. 
Table 4 Tweet sentiment obtained by manual and automated classifications

\begin{tabular}{|c|c|c|c|c|c|c|}
\hline & Positive (manual) & Negative (manual) & Neutral (manual) & Uncertain (manual) & Discard (manual) & \\
\hline Positive (automated) & 559 & 95 & 871 & 4 & 134 & 1,663 \\
\hline Negative (automated) & 69 & 286 & 384 & 6 & 93 & 838 \\
\hline Neutral (automated) & 751 & 513 & 3,321 & 123 & 440 & 5,148 \\
\hline \multirow[t]{2}{*}{ Discard (automated) } & 57 & 82 & 158 & 5 & 49 & 351 \\
\hline & 1,436 & 976 & 4,734 & 138 & 716 & 8,000 \\
\hline
\end{tabular}

\subsection{Measures of Diffusion Volume and Speed}

We obtained the number of retweets for each tweet and $N$ retweet time as measures of diffusion volume and speed, respectively. Each retweet had a timestamp and the ID of the original tweet. For each original tweet, $T$, we counted the number of retweets of $T$. We obtained the $N$-retweet time of $T$ by calculating the interval between the time $T$ was posted and the time the $N$ th retweet was posted.

\subsection{Methods for Statistical Analysis}

To begin with, we examined the mean and distribution of the measures of message virality for the message sentiments. We classified all tweets into positive, negative, or neutral. For each category, we obtained the mean and distribution of the number of retweets and $N$-retweet time. When analyzing dataset $D_{S}$, we estimated the mean of the number of retweets in the population, given that dataset $D_{S}$ was obtained from dataset $D_{A}$ by biased sampling. The method of estimating the mean number of retweets of positive tweets was as follows. Let $\mu_{i}^{p}$ be the sample mean of the number of retweets of positive tweets in section $i$ (Table 1) and in dataset $D_{S}$, and let $f_{i}^{p}$ be the number of positive tweets in section $i$ and in dataset $D_{A}$ divided by the number of positive tweets in dataset $D_{A}$. The mean number of retweets of positive tweets was estimated as $\sum_{i} f_{i}^{p} \mu_{i}^{p}$.

Next, we performed regression analysis to investigate the effects of message sentiment on virality, taking into consideration other factors related to retweet behavior. We used the variables shown in Table 5. Following the method in (Stieglitz and Dang-Xuan, 2013), we used the presence of URLs, hashtags, and the number of followers as control variables because these factors affect message diffusion (Stieglitz and Dang-Xuan, 2013; Suh et al., 2010; Naveed et al., 2011). Using these control variables, we examined the effects of message sentiment on virality, eliminating the effects of other factors. We did not include a variable for the activity of twitter users because this did not affect message diffusion (Stieglitz and Dang-Xuan, 2013). We did not use dataset $D_{S}$ for regression analysis because it was obtained from a biased sampling.

Following the method in (Stieglitz and Dang-Xuan, 2013), we used a binomial regression model for regression
Table 5 Variables used in regression analysis

\begin{tabular}{l|l}
\hline \hline Variable & Description \\
\hline RTnum & Number of retweets \\
NRTtime & Time interval between original tweet and N th retweet \\
pos & Categorical variable showing tweet is positive \\
neg & Categorical variable showing tweet is positive \\
follower & Number of followers \\
URL & Categorical variable for whether tweet includes URL \\
hash & Categorical variable for whether tweet includes hashtag \\
\hline
\end{tabular}

of RTnum because the variance of the number of retweets was high (Tables 1 and 2). In the negative binomial regression model, the relation between dependent and independent variables was modeled as

$$
\begin{aligned}
\log (\text { RTnum })= & \beta_{0}+\beta_{1} \text { URL }+\beta_{2} \text { hash } \\
& +\beta_{3} \log (\text { follower })+\beta_{4} \text { pos }+\beta_{5} \text { neg } \\
\text { RTnum }= & e^{\beta_{0}} \times e^{\beta_{1} U R L} \times e^{\beta_{2} \text { hash }} \\
& \times \text { follower }^{\beta_{3}} \times e^{\beta_{4} \text { pos }} \times e^{\beta_{5} \text { neg }}
\end{aligned}
$$

where $\beta_{n}$ is the regression coefficient. Note that follower is log-transformed because the distribution of the number of followers is heavy-tailed. For the regression of NRTtime, we used a simple linear regression model.

\section{Results of Analyses}

\subsection{Negative messages spread widely}

To investigate the relation between message sentiment and diffusion volume, we examined the mean number of retweets for each category based on tweet sentiment (Fig. 1). The bars on the left-hand side of the figure show the results obtained from dataset $D_{S}$, and the bars on the righthand side show the results obtained from dataset $D_{A}$. The results of dataset $D_{S}$ show estimated mean values that are explained in Section 3.5.

Figure 1 shows that the retweet volume of negative tweets is approximately $20-60 \%$ higher than that of neutral tweets, and the retweet volumes of positive and neutral tweets are similar to each other. We performed the pairwise test on the results of dataset $D_{A}$ using the Steel-Dwass method (Steel, 1960; Dwass, 1960), and discovered that 


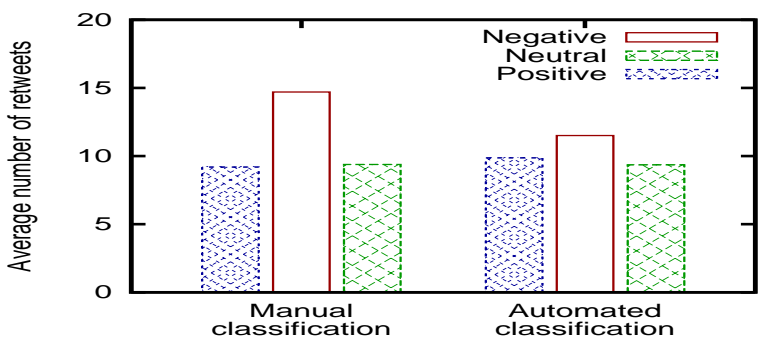

Fig. 1 Relation between tweet sentiment and mean number of retweets. Left-hand bars show estimated mean values obtained from dataset $D_{S}$, and right-hand bars show simple mean values obtained from dataset $D_{A}$. Population is tweets whose number of retweets is greater than one. Retweet volume of negative tweets is higher than for positive and neutral tweets.

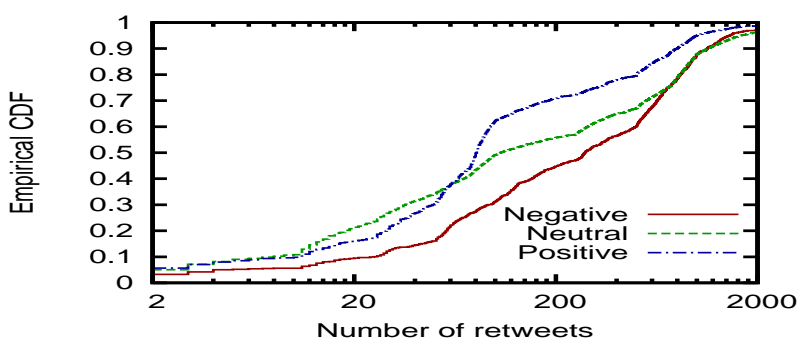

(a) Manual classification

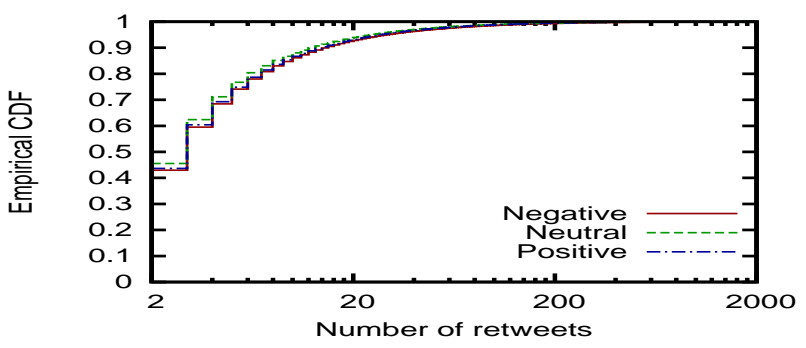

(b) Automated classification

Fig. 2 Cumulative distribution of number of retweets for each category. Note that (a) shows cumulative distributions of retweet volume for sampled tweets, not total population. Negative tweets tend to have higher retweet volume than positive and neutral tweets.

there were significant differences in the number of retweets between any two categories based on sentiment $(p<0.05)$. These results suggested that the retweet volume of negative tweets was higher than that of neutral and positive tweets, and the retweet volume of positive tweets was similar to that of neutral tweets. The differences of the mean values obtained with datasets $D_{S}$ and $D_{A}$ may have been caused by the difference between the automated and manual classifications (Table 4).

Next, we investigated the distributions of the number of retweets for each category (Fig. 2). Figure 2 confirms that negative tweets tend to have a higher retweet volume than positive and neutral tweets. We also find that positive tweets tend to have a slightly higher retweet volume than neutral tweets (Fig. 2 (b)).
Table 6 Negative binomial regression results for RTnum. ***: significant at $1 \%$ level, **: significant at $5 \%$ level, *: significant at $10 \%$ level.

\begin{tabular}{lrr}
\hline \hline \multicolumn{3}{c}{ Dependent variable: $R$ Rnum } \\
\hline & & \\
Independent variables & Coeff. $\beta$ & $e^{\beta}$ \\
\hline pos $^{* * *}$ & 0.131 & 1.139 \\
neg*** & 0.311 & 1.365 \\
$\log (\text { follower })^{* * *}$ & 0.203 & \\
URL $L^{* * *}$ & 0.546 & 1.726 \\
hash * $^{* *}$ & 0.291 & 1.338 \\
constant*** & 0.467 & \\
& & \\
\hline Pseudo $R^{2}$ & & 0.030 \\
Num. of observations & & $4,137,064$ \\
\hline
\end{tabular}

Next, we performed regression analysis to investigate the relation between message sentiment and virality, eliminating the effects of other factors affecting message diffusion. We performed negative binomial regression analysis for investigating the effects of message sentiment on diffusion volume. The dependent variable was RTnum, and the independent variables were pos, neg, follower, URL, and hash. Table 6 shows the regression analysis results. The regression coefficient, $\beta$, and the values of $e^{\beta}$ for each variable are shown in the table to demonstrate the effects of each independent variable on the dependent variable.

The result of the regression analysis showed that whether the sentiment of a tweet was negative or positive, this factor increased the number of its retweets in the model. The strength of the effect of each variable could be estimated on the basis of the regression coefficient, $e^{\beta}$ (Eq.(2)). The regression coefficient of neg suggested that negative tweets were retweeted $36.5 \%$ more often than neutral tweets, consistent with previous results. This indicated that negative sentiment was a major driving factor for tweet diffusion, because the regression coefficient of neg was comparable with that of hash, which was a major driving factor for retweets (Suh et al., 2010; Naveed et al., 2011). In addition, positive sentiment in a tweet also increased retweet volume, although the effect was weaker than that of other factors. In summary, this result showed that negative sentiment was a strong driving factor for retweet diffusion, and that positive sentiment was not a strong driving factor for retweet diffusion, although it also slightly affected diffusion volume.

Note that the value of pseudo $R^{2}$ in our model is low. Message diffusion in social media is often difficult to explain, and there are many other driving factors. In this analysis, we can conclude that the effects of negative and positive sentiment are statistically significant, and the effect of negative sentiment is similar to that of hashtags. We do not claim that we can model the retweet volume only using these variables. We also note that the value of pseudo $R^{2}$ in our model is lower than that obtained in (Stieglitz and DangXuan, 2013). The reason is that our dataset does not in- 


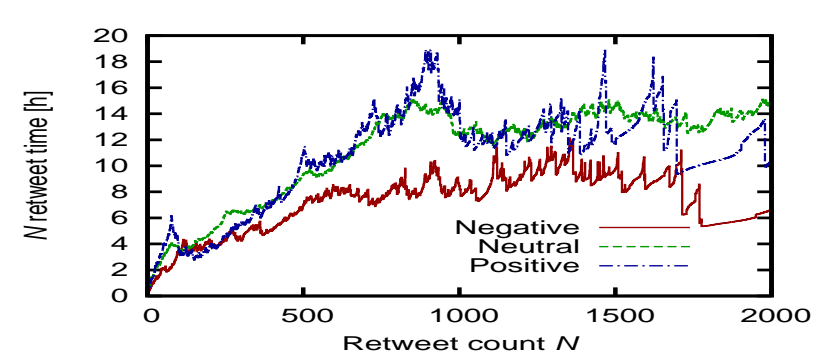

(a) Manual classification

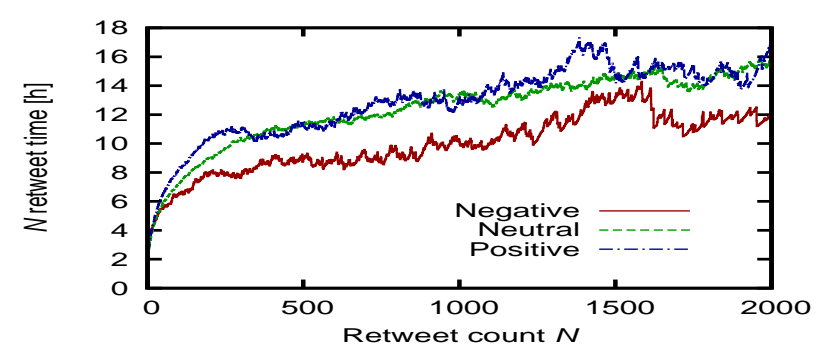

(b) Automated classification

Fig. 3 Average $N$-retweet time per category. Average $N$-retweet time of negative tweets is shorter than those of positive and neutral tweets.

clude tweets that are not retweeted. URLs and hashtags in tweets are strong factors affecting whether the tweets are retweeted (Suh et al., 2010; Naveed et al., 2011). Therefore, we can generally construct a more accurate model explaining RTNum on the basis of these independent variables if the dataset includes tweets with no retweet than if the dataset only includes tweets with more than one retweet.

\subsection{Negative messages spread rapidly}

Next, we dealt with retweet speed by using average $N$ retweet time. Figure 3 shows the average $N$-retweet times for each category. The average $N$-retweet time was obtained by calculating the average $N$-retweet time for tweets that were retweeted at least $N$ times. Because the number of samples with a high retweet count, $N$, was limited, the average values fluctuated if $N$ was high.

Figure 3 shows that the average $N$-retweet time of negative tweets is shorter than those of positive and neutral tweets. In particular, when $N>100$, the average $N$-retweet time of negative tweets is approximately $20 \%$ shorter than those of positive and neutral tweets (i.e., negative tweets spread $25 \%$ faster than positive and neutral tweets). Note that the fraction of tweets retweeted more than 100 times is only $1 \%$ of the collected dataset. Namely, tweets with a retweet count of $N>100$ have high virality in terms of diffusion volume. These results suggest that negative tweets spread faster than positive and neutral tweets, particularly for tweets with a high diffusion volume. In contrast, the $N$ - retweet time of positive tweets was slightly longer than that of neutral tweets.

We investigated the distribution of $N$-retweet time of tweets for each category. Figures 4 , and 5 show the cumulative distributions of $N$-retweet time for each category when $N=100$ and $N=1000$, respectively. We chose $N=100$ to investigate the diffusion speed of tweets with high diffusion volume, and chose $N=1000$ to investigate the diffusion speed of tweets with quite high diffusion volume. Note that in terms of diffusion volume, tweets with a retweet count of $N>100$ are approximately top $1 \%$ tweets and those with a retweet count of $N>1000$ are approximately top $0.05 \%$ tweets.

These results show that negative tweets spread faster than neutral and positive tweets if the retweet count, $N$, is quite high. Figure 5 shows that the diffusion speed of negative tweets is faster than that for tweets with other sentiments when $N=1000$. In contrast, Figure 4 shows that the $N$ retweet time for negative tweets and tweets with other sentiments are similar. The difference in $N$-retweet time between positive and neutral tweets was only observed in Fig. 4(b). The pairwise test with the Steel-Dwass method (Steel, 1960; Dwass, 1960) showed that there was a significant difference in 100-, and 1000-retweet time among tweet sentiment categories in dataset $D_{A}(p<0.05)$.

Next, we examined the relation between message sentiment and diffusion speed by using regression analysis. We used 100-RTtime and 1000-RTtime as dependent variables. In addition to the independent variables used in the diffusion volume regression analysis, we used RTnum as an independent variable. The reason is that tweets with a high diffusion volume are considered to spread fast, and therefore, RTnum may affect 100-RTtime and 1000-RTtime. In the following analyses, a linear regression model was used. Tables 7 and 8 show the regression results for the dependent variables of 100-RTtime, and 1000-RTtime, respectively. Standardized coefficients of variables are shown in the tables for comparing the variables of different scales.

Table 8 indicates that the presence of negative sentiment in a message decreased the 1000-retweet time $(p<0.1)$. The regression coefficient of neg suggests that 1000-retweet time of negative tweets is approximately 1.9 hour shorter than neutral tweets. This result was consistent with the observation in the previous results that negative tweets spread faster when the number of retweets is quite high. Table 7 shows that the presence of negative sentiment in a message did not significantly affect 100 -retweet time. This result suggests that negative sentiment does not have a significant effect on diffusion speed when the diffusion volume is not quite high. Observing other control variables, we find, as intuitively expected, that follower, URL, and RTnum significantly affect diffusion speed, and standardized coefficients suggest that particularly RTnum has strong effects on both 


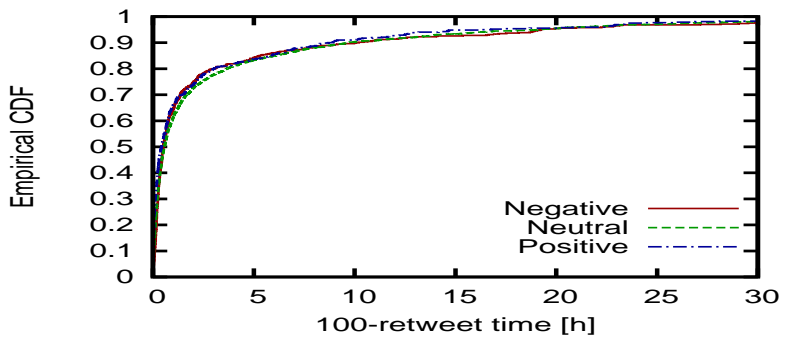

(a) Manual classification

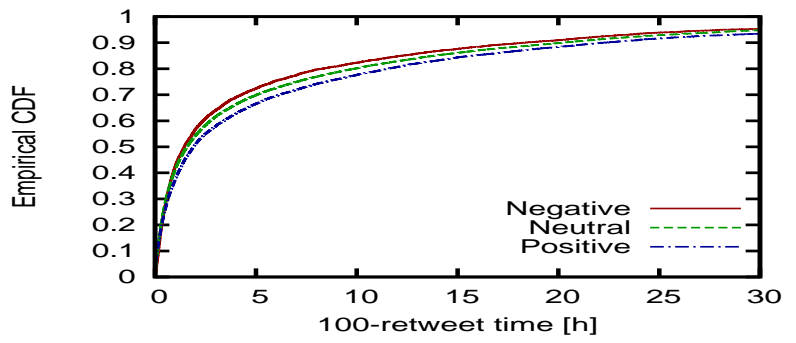

(b) Automated classification

Fig. 4 Cumulative distribution of 100-retweet time for each category. 100-retweet times for negative tweets and tweets with other sentiments are similar.

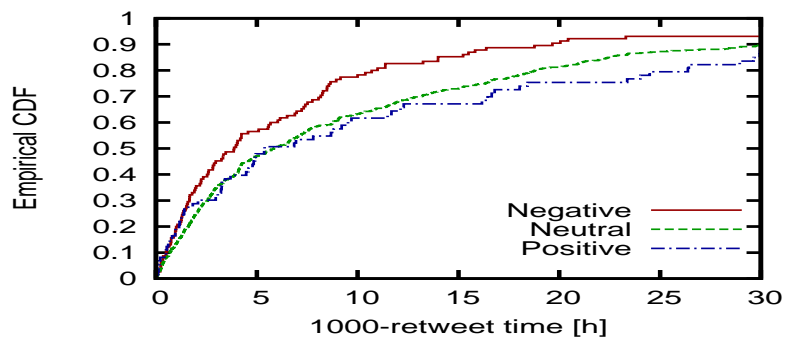

(a) Manual classification

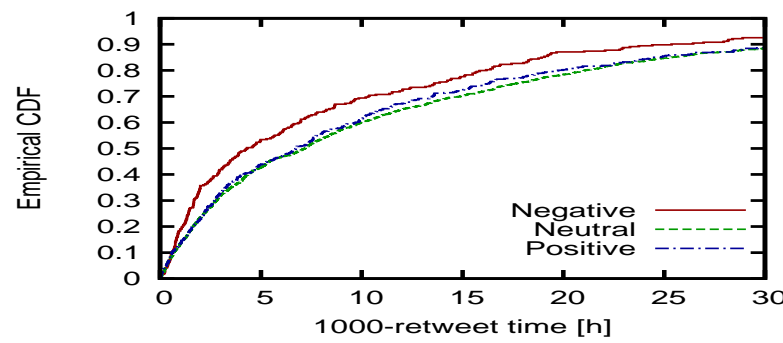

(b) Automated classification

Fig. 5 Cumulative distribution of 1000-retweet time for each category. Diffusion speed of negative tweets is faster than that of tweets with other sentiments when $N=1000$.

Table 7 Regression results for 100-RTtime[h]. ***: significant at $1 \%$ level, **: significant at $5 \%$ level, *:significant at $10 \%$ level.

\begin{tabular}{|c|c|c|}
\hline \multicolumn{3}{|c|}{ 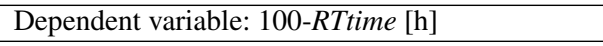 } \\
\hline Independent variables & Coeff. $\beta$ & Std. Coeff. \\
\hline pos*** & 1.149 & 0.032 \\
\hline neg & 0.052 & 0.001 \\
\hline $\log (\text { follower })^{* * *}$ & -0.632 & -0.113 \\
\hline$U R L^{* * *}$ & 1.889 & 0.062 \\
\hline hash*** & 1.992 & 0.056 \\
\hline RTnum*** & -0.003 & -0.126 \\
\hline constant $* * *$ & 11.855 & \\
\hline$R^{2}$ & 0.040 & \\
\hline Num. of observations & 48,814 & \\
\hline
\end{tabular}

of 100-retweet time and 1000-retweet time. Table 7 indicates that the presence of positive sentiment in a message increased the 100-retweet time $(p<0.05)$, which is consistent with previous results (Figs. 3(b) and 4(b)).

\subsection{Negative messages do not spread recurrently}

Finally, we focus on the relation between temporal patterns of tweet diffusion and tweet sentiment using one-year records of retweets, datasets $D_{A L}$ and $D_{S L}$. During our analysis, we discovered that while most tweets spread over a period of a day or a few days, there existed long-lived tweets
Table 8 Regression results for 1000-RTtime[h]. ***: significant at $1 \%$ level, **: significant at 5\% level, *: significant at $10 \%$ level.

\begin{tabular}{|c|c|c|}
\hline \multicolumn{3}{|c|}{ 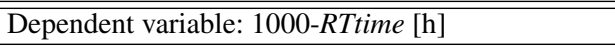 } \\
\hline Independent variables & Coeff. $\beta$ & Std. Coeff. \\
\hline pos & 0.941 & 0.021 \\
\hline neg* & -1.922 & -0.036 \\
\hline $\log (\text { follower })^{* *}$ & -0.331 & -0.048 \\
\hline$U R L^{* * * *}$ & 5.055 & 0.134 \\
\hline hash & 0.339 & 0.007 \\
\hline RTnum*** & -0.002 & -0.247 \\
\hline constant*** & 17.365 & \\
\hline$R^{2}$ & 0.080 & \\
\hline Num. of observations & 2,194 & \\
\hline
\end{tabular}

whose diffusion lasted for several months. Figure 6(a) shows an example of a typical diffusion for a tweet; i.e., a series of changes in the number of retweets over time. Typical tweets spread as a single burst, and diffusion did not last over a couple of days. In contrast, we discovered that there existed recurrent tweet diffusion patterns. Figure 6(b) shows an example of recurrent tweet diffusion. On a popular social medium, Facebook, recurrence of message diffusion had recently been observed (Cheng et al., 2016), and we discovered that such recurrent cascades of repostings had also been observed on Twitter.

Our question here is how the temporal diffusion pattern of messages is affected by sentiment. To perform a quanti- 
Table 9 Summary of findings

\begin{tabular}{|c|c|c|}
\hline & Conclusion & Supporting results \\
\hline Retweet volume & $\begin{array}{l}\text { Negative is higher than positive and neutral } \\
\text { Positive is slightly higher than neutral }\end{array}$ & $\begin{array}{l}\text { Figs. } 1,2 \text {, and Tab. } 6 \\
\text { Tab. } 6\end{array}$ \\
\hline Retweet speed & $\begin{array}{l}\text { Negative is faster than positive and neutral } \\
\text { for quite high diffusion volume } \\
\text { Neutral is slightly faster than positive } \\
\text { for not quite high diffusion volume }\end{array}$ & $\begin{array}{l}\text { Figs. } 3,5 \text {, and Tab. } 8^{*} \\
\text { Figs. 3(b), 4(b), and Tab. } 7\end{array}$ \\
\hline Recurrence & Negative is less frequent than positive and neutral & Fig. 7 \\
\hline
\end{tabular}

* Evidence in Table 8 is not so strong, but supports this conclusion.

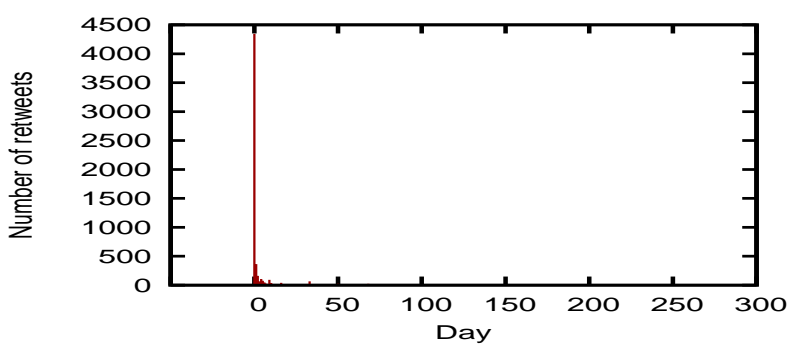

(a) Diffusion with single burst

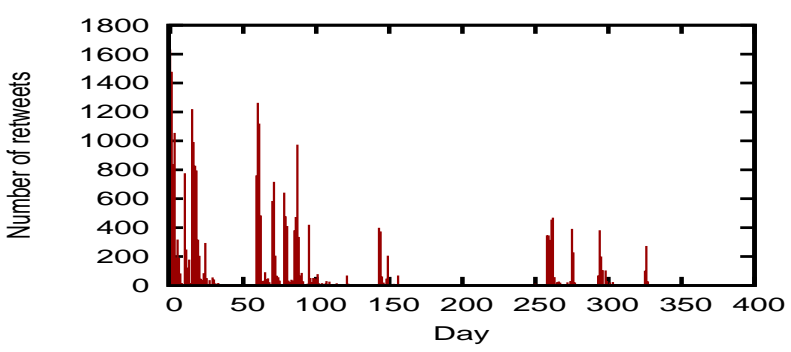

(b) Recurrent diffusion

Fig. 6 Examples of temporal patterns of tweet diffusion. Typical tweets are limited to single-burst spread, and their diffusion only continues for some days. Some tweets spread with multiple bursts.

tative investigation, we counted the number of tweets that spread recurrently for positive, negative, and neutral tweets. Following Cheng et al. (2016), we used their definition of recurrence, explained as follows. Consider a tweet diffusion over $t$ days. We counted the number of peak days of the tweet diffusion. Let $r_{i}$ be the number of retweets observed on day $i$. Day $i$ is called a peak day if $r_{i}$ is at least $h 0$, at least $m$ times the mean number of retweets per day, and a local maximum in $[i-w, i+w]$ days. Finally, between any two adjacent peak days $p_{i}$ and $p_{i+1}$, the number of retweets must fall below $v \cdot \min \left\{r_{p_{i}}, r_{p_{i+1}}\right\}$. If a diffusion of a tweet has multiple peaks, the tweet diffusion is considered to be recurrent. We used $h_{0}=10, m=2, w=7$, and $v=0.5$, which were the same parameters used in (Cheng et al., 2016).

We examined the fraction of tweets with recurrent diffusion for each category, based on tweet sentiment (Fig. 7). The three bars on the left show the results obtained from dataset $D_{S L}$, and the three bars on the right show the results

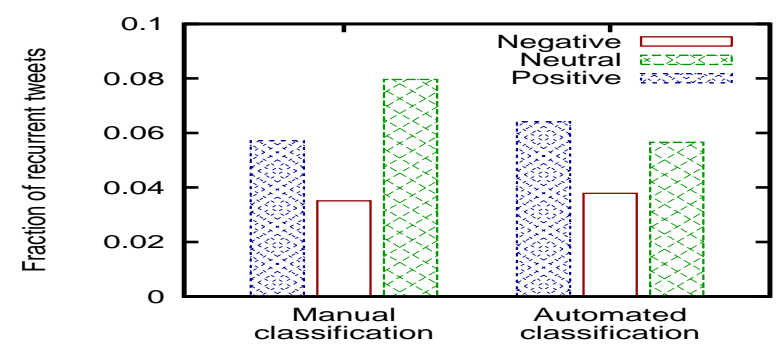

Fig. 7 Relation between tweet sentiment and fraction of recurrently spreading tweets. Left-hand bars show result for dataset $D_{S L}$, and right-hand bars show result for dataset $D_{A L}$. Population is tweets whose number of retweets is greater than 100 . Fraction of recurrent diffusion of negative tweets is lower than those of positive and neutral tweets.

obtained from dataset $D_{A L}$. Note that tweets with more than 100 retweets were used in this analysis in order to eliminate tweets with low diffusion volume.

Figure 7 shows that the fraction of recurrent diffusion of negative tweets is lower than those of positive and neutral tweets. We performed the Chi-squared test on the results of dataset $D_{A L}$, and discovered that there were significant differences in the fraction of recurrent tweets between any two categories based on sentiment $(p<0.05)$. These results suggest that recurrent diffusion of negative messages is less frequent than those of positive and neutral messages.

These results are consistent with the findings in the analyses on diffusion volume and speed. As discussed in (Cheng et al., 2016), if the virality of a message is strong, the message can reach its audience in a single burst. Therefore, it is considered to be natural that negative messages, which tend to be viral, are not likely to spread recurrently.

Our findings are summarized in Table 9. We conclude that negative tweets spread more widely than positive or neutral tweets, and suggest that negative tweets spread faster than tweets with other sentiments, particularly for tweets with a quite high diffusion volume. As a result of their high virality, negative messages have less frequent recurrent spreading than positive and neutral messages. The effect of positive sentiment is weaker than that of negative sentiment, although positive tweets tend to be retweeted slightly more than neutral tweets. 


\section{Model-based explanation}

\subsection{A simple model of message diffusion}

We present a simple message diffusion model, called "independent cascade with Zipf-distributed delay" (IC-ZD), that can reproduce the characteristics observed in this paper. The IC-ZD model can produce message diffusion with both single and multiple bursts. In the IC-ZD model, the virality of diffusion of a message can be changed by parameteradjustment.

The IC-ZD model is based on a popular influence cascade model, called "independent cascade" (IC) (Kempe et al., 2003). The IC model is a model for influence cascade on a graph. In the IC model, each active node has a single opportunity to spread its influence to adjacent nodes with a predefined influence spread probability $p$. We consider influence in the IC model as a message, and an active node in the IC model as a user who has retweeted the message. The parameter $p$ controls diffusion volume. Using the IC model, in which each node has a single opportunity to spread influence, is considered to be a natural approach to modeling tweet diffusion, in which each user can repost a tweet only once. However, in the original IC model, when node $i$ is activated at time-step $t$, node $i$ spreads its influence to an adjacent inactive node at the next time-step $t+1$, and thus cannot produce a recurrent diffusion pattern.

To produce a recurrent diffusion pattern, the IC-ZD model incorporates time-delay for influence (message) diffusion. In the IC-ZD model, when node $i$ is activated at timestep $t$, it has a single opportunity to spread influence to an adjacent inactive node $j$ with a probability $p$ at time-step $t+\delta$, where $\delta$ is a random variable obtained from a Zipf distribution with power-law exponent $\gamma$. The parameter $\gamma$ controls diffusion speed. Since bursty behaviors are characterized by long-tailed distributions of inter-event time (Karsai et al., 2012; Zhao et al., 2011), and in our data, the distribution of time intervals between retweets follows a powerlaw (see Fig. 8), we expect that power-law time delays of message diffusion can reproduce bursty message diffusions. Note that the IC model with time delay is not a novel model, since it was also used in (Liu et al., 2012). However, we use Zipf distribution for time delay, whereas Liu et al. (2012) used Poisson distribution.

\subsection{Simulation setups and results}

We conducted simulations using the IC-ZD model. Unfortunately, since the social network structure of users in our retweet dataset was not available, we used a publicly available social network data of Twitter users (De Domenico et al., 2013). The data contain retweet relationships among 256,491 users. On the network, we performed simulations

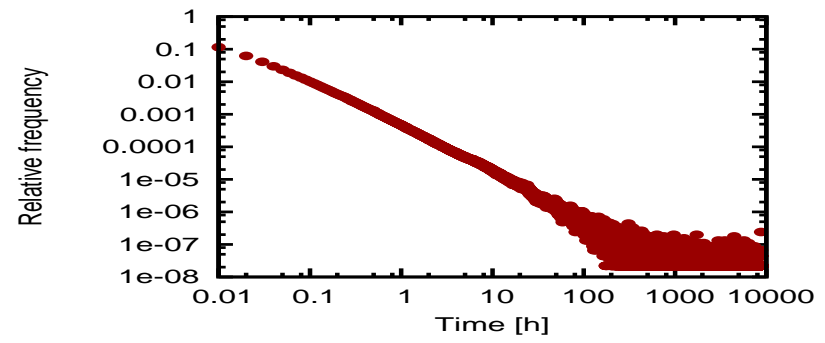

(a) Distribution of time interval between retweets

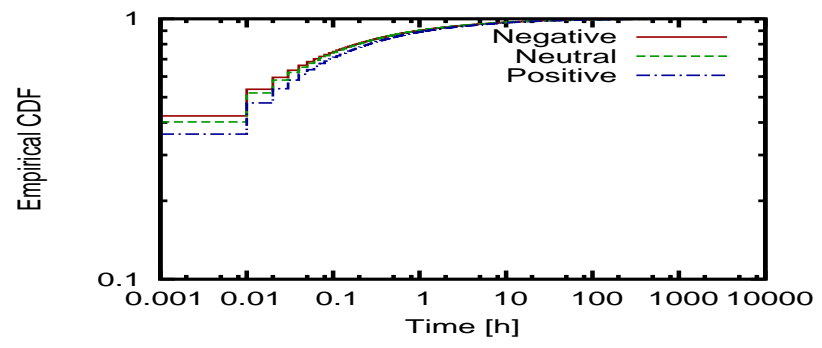

(b) CDF of time interval between retweets for each category

Fig. 8 Distribution of time interval between adjacent two retweets in dataset $D_{A L}$. Time interval between retweets follows power-law.

using the model, activating a randomly selected node at time-step 0 . We varied influence spread probability, $p$, between 0.0050 and 0.0080 , and parameter of time delay, $\gamma$, between 1.80 and 2.00. For each parameter setting, we performed 50,000 simulation runs.

Examples of temporal patterns of message diffusion in the model are shown in Fig. 9. These figures confirm that our model can reproduce message diffusion for both single burst and recurrent bursts.

Figure 10 shows the average $N$-retweet time obtained from 50,000 simulation runs for parameter settings (a) $p=$ $0.0068, \gamma=1.81$; and (b) $p=0.0070, \gamma=1.86$. Here, $N$-retweet time for a simulation run is defined as the timestep where the number of active nodes exceeds $N$ for the first time in the simulation run. On the basis of this figure, we discover that $N$-retweet time for parameter setting (b) is shorter than that for parameter setting (a), particularly when the retweet count $N$ is high. Let us assume that the simulations with parameter setting (a) simulate positive tweet diffusion, and the simulations with parameter setting (b) simulate negative tweet diffusion. Then, we find that the model can reproduce the characteristics observed in Section 4.2 showing that negative messages spread faster, particularly when the diffusion volume is high. Here, fractions of recurrent diffusion in diffusion where size is greater than 100 are $4.0 \%$ for parameter (a) and $2.7 \%$ for parameter (b), and the average diffusion volume is 6.8 for parameter (a) and 10.7 for parameter (b). These results also indicate that the model can simulate tweet diffusion with different viralities, and can reproduce the characteristics shown in this paper. 


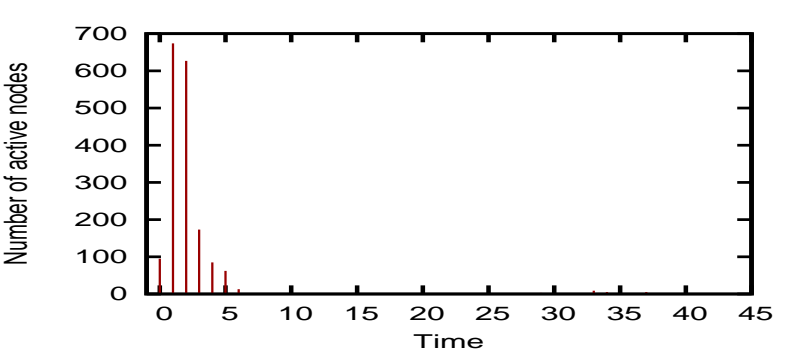

(a) Diffusion with single burst

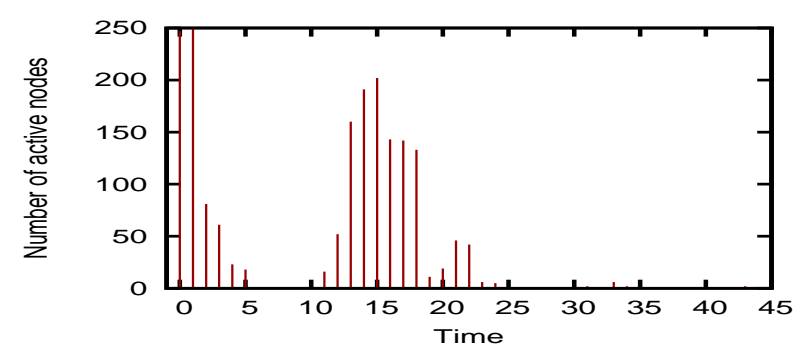

(b) Recurrent diffusion

Fig. 9 Examples of temporal patterns of message diffusion generated by IC-ZD model. One unit of time shown in these figures corresponds to 24 steps in simulations.

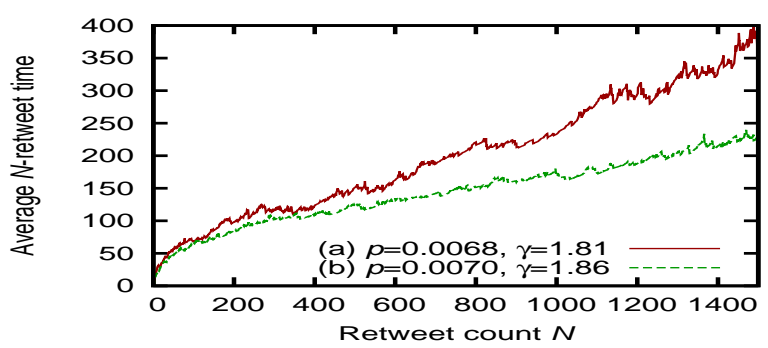

Fig. $10 N$-retweet time obtained from simulations for two parameter settings: (a) $p=0.0068, \gamma=1.81$; and (b) $p=0.0070, \gamma=1.86$. Assuming (a) as positive tweet diffusion and (b) as negative tweet diffusion, we discover that this model can reproduce characteristics shown in Fig. 3 demonstrating that negative messages spread faster, particularly when diffusion volume is high.

Finally, we conducted simulations on the same network using the original IC model (Kempe et al., 2003), and the IC model with Poisson-distributed delay (IC-PD) (Liu et al., 2012). We varied the influence-spread probability $p$ between 0.0050 and 0.0080 for the IC and IC-PD models, and the average delay $\lambda$ between 1 and 50 for the IC-PD model. For each parameter setting, we performed 50,000 simulation runs. The results of the simulation runs using the IC model did not show a recurrent diffusion pattern. As we discussed in the previous subsection, long-term diffusion cannot be produced using the IC model, since nodes spread influence to their neighbors immediately after they become active in the IC model. In contrast, in the IC-PD model, although long-term recurrent diffusion can be observed when parameter $\lambda$ is high, bursty short-term diffusion cannot. Figure 11 shows cumulative distributions of 100-retweet time obtained

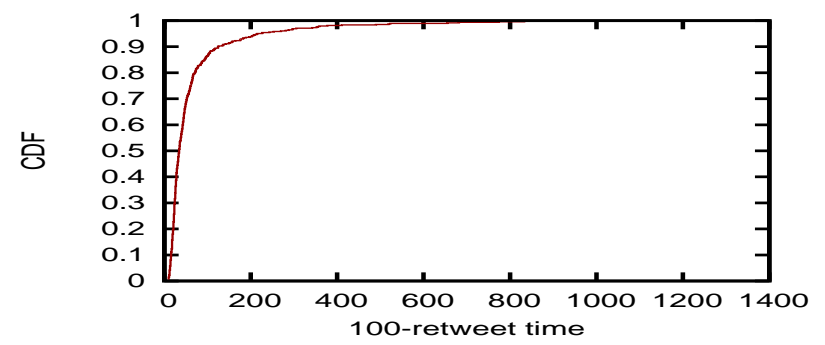

(a) IC-ZD model

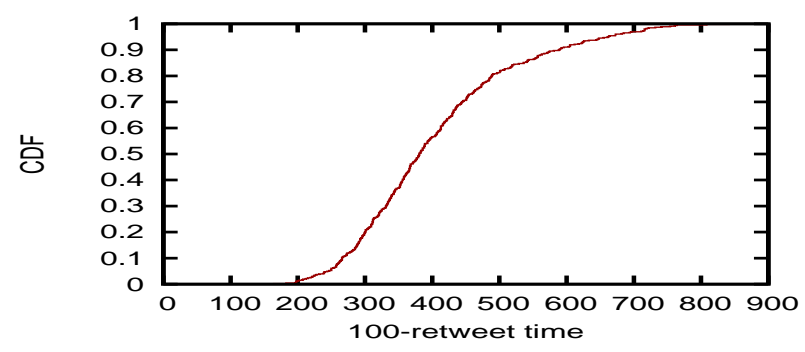

(b) IC-PD model

Fig. 11 Cumulative distribution of 100-RT time. (a) IC-ZD model with $p=0.0070$ and $\gamma=1.86$, and (b) IC-PD model with $p=0.0070$ and $\lambda=35$. IC-ZD model and IC-PD model with these parameter settings produce similar diffusion volumes and fractions of recurrent diffusion. IC-ZD model can successfully reproduce form of distribution of 100-retweet time similar to that observed in this paper, whereas IC-PD model cannot.

from simulations using the IC-ZD model $(p=0.0070$ and $\gamma=1.86)$ and the IC-PD model ( $p=0.0070$ and $\lambda=35$ ). Note that the IC-ZD and IC-PD models with these parameter settings produce similar diffusion volumes (10.7 and 10.1) and fractions of recurrent diffusion $(2.7 \%$ and $2.8 \%)$. Figure 11 shows that the IC-ZD model can successfully reproduce a form of distribution similar to that shown in Fig. 4, whereas the IC-PD model cannot. These results suggest that the delay with Zipf distribution used in our proposed model is a key to reproducing the characteristics of tweet diffusion.

\section{Discussion}

\subsection{Findings and implications}

Our study shows that negative tweets are more viral than positive tweets in terms of retweet volume. This is strong evidence of existence of negativity bias (Taylor, 1991; Baumeister and Bratslavsky, 2001; Rozin and Royzman, 2001) on social media. As discussed in Section 2, prior work by Stieglitz and Dang-Xuan (2013) only partially supported the negativity bias, and that by Gruzd et al. (2011) showed opposite results. These studies targeted domainspecific tweets, and as discussed in (Stieglitz and DangXuan, 2013), the tweet domain altered how tweet sentiment affected virality. However, our study investigates the effects 
of tweet sentiment using non-domain-specific tweets. Consequently, our study shows that negative tweets are generally more viral than positive tweets, indicating negativity bias on social media.

The results for retweet speed also partly support the negativity bias. We investigated the relation between tweet sentiment and $N$-retweet time. For a quite high retweet count, $N$, negative tweets spread faster than positive and neutral tweets. Stieglitz and Dang-Xuan (2013) and Ferrara and Yang (2015) used only 1-retweet time, and discovered that there was no significant difference in retweet speed between positive and negative tweets. Our study shows that negative tweets spread faster than positive tweets when the diffusion volume is quite high. To the best of our knowledge, ours is the first study to show the effects of sentiment on diffusion speed for tweets with a high diffusion volume.

Our results also show that the effects of positive sentiment in a tweet on its virality are weak. This contradicts the results in (Stieglitz and Dang-Xuan, 2013, 2012; Gruzd et al., 2011; Naveed et al., 2011; Ferrara and Yang, 2015), suggesting that positive and negative sentiment in a message increase its virality. One possible cause of the difference between our study and previous studies might be the nationality of the subject audience. Ours is the first study to use Japanese tweets to investigate the relation between tweet sentiment and virality. The linguistic and cultural differences may affect the results because usage patterns for Twitter users differ across languages (Hong et al., 2011a). However, more analyses are necessary to reveal the causes of these discrepancies.

Our longitudinal analysis of temporal patterns of message diffusion shows that some messages spread recurrently, and also demonstrated that the IC model with power-law time delay could produce such recurrent patterns of message diffusion. Cheng et al. (2016) also proposed a model of recurring message diffusion, but the model assumed multiple copies of a message. To the best of our knowledge, our study is the first to present a model of recurrent message diffusion that assumes a single copy.

Our results have several implications. First, it is important for companies to address negative opinions about their products on social media. Even if the numbers of users with positive and negative opinions are equal, negative opinions may spread faster and further, thus reaching a higher number of people than the positive opinions. Second, it is important to track the sentiment of individual tweets to prevent unintentional tweet diffusion. Recently, negative rumors and misinformation spreading on social media, known as flaming, have posed serious problems, and blocking rumor spread is of interest to researchers (Budak et al., 2011; Wen et al., 2014). Our results suggest that individual users should take care to avoid unnecessary negative terms to prevent unintentional information spread. A mechanism to de- tect and alert users to tweet sentiment may be an effective approach. Third, the proposed IC-ZD model can be used for analyzing message diffusion on social media with respect to several criteria. For instance, the model could be useful for analyzing the effects of network structure on the message diffusion, and the effects of increase in virality on diffusion volume and speed.

\subsection{Limitations}

We used a large-scale dataset including 4.1 million tweets, which was merely a sample of messages on social media. We studied Twitter as a social media platform, and only analyzed Japanese tweets. We chose Twitter because of the availability of large-scale Twitter tweet data; however, to generalize the results, it was necessary to analyze data from other platforms. Most previous studies used English tweets (Gruzd et al., 2011; Naveed et al., 2011; Hansen et al., 2011), and some used German tweets (Stieglitz and DangXuan, 2013, 2012), whereas our study used Japanese tweets. Our study showed that for Japanese tweets, tweet sentiment was a major driving factor for retweets. However, the research methodologies of this study were different from those of previous studies, particularly regarding tweet topics, and Twitter usage patterns are different across languages (Hong et al., 2011a). Therefore, the differences among different languages should be investigated. In order to examine how to generalize our results, we are also interested in a number of related tasks, such as expanding the data collection period, and investigating messages during social events (e.g., national festival holidays).

We used a simple approach for automated classification of large-scale tweets based on their sentiment (Hansen et al., 2011; Naveed et al., 2011). Although we obtained similar results from datasets constructed by automated and manual classifications, use of a more sophisticated method to determine tweet sentiment may produce better results. Because each tweet is short, it is difficult to determine tweet sentiment, and there have been several studies regarding how to determine tweet sentiment accurately (Barbosa and Feng, 2010; Agarwal et al., 2011; Kontopoulos et al., 2013; Gonçalves et al., 2013). In future work, we intend to apply these techniques to our dataset, and To validate the results in this paper.

\section{Conclusion}

We investigated the relation between the sentiment of a tweet and its virality in terms of diffusion volume and speed by analyzing 4.1 million tweets on Twitter. We used the number of retweets and $N$-retweet time as measures of tweet virality. We discovered that negative tweets spread more 
widely than positive and neutral tweets, and that negative tweets spread faster than either positive or neutral tweets when the diffusion volume was quite high. We showed that the diffusion volume of negative tweets was 20-60\% higher than that of positive and neutral tweets, and that the diffusion speed of negative tweets was $25 \%$ faster than that of positive and neutral tweets when the diffusion volume was quite high. We also conducted a longitudinal analysis of tweet diffusion, and discovered that recurrent diffusion of negative messages was less frequent than that of positive and neutral tweets. Moreover, we presented a simple tweet diffusion model, the IC-ZD model, that can reproduce the characteristics of tweet diffusion observed in this paper.

\section{Acknowledgements}

The authors would like to thank Dr. Mitsuo Yoshida of Toyohashi University of Technology for his support to the data collection, and Hisayuki Mori of Kwansei Gakuin University for helping the analyses. This work was partly supported by the Telecommunications Advancement Foundation.

\section{References}

Agarwal A, Xie B, Vovsha I, Rambow O, Passonneau R (2011) Sentiment analysis of Twitter data. In: Proceedings of the Workshop on Languages in Social Media (LSM'11), pp 30-38

Bakshy E, Hofman JM, Mason WA, Watts DJ (2011) Everyone's an influencer: Quantifying influence on Twitter. In: Proceedings of the 4th ACM International Conference on Web Search and Data Mining (WSDM'11), pp 65-74

Bakshy E, Rosenn I, Marlow C, Adamic L (2012) The role of social networks in information diffusion. In: Proceedings of the 21st International Conference on World Wide Web (WWW'12), pp 519-528

Barbosa L, Feng J (2010) Robust sentiment detection on Twitter from biased and noisy data. In: Proceedings of the 23rd International Conference on Computational Linguistics (COLING'10), pp 36-44

Baumeister RF, Bratslavsky E (2001) Bad is stronger than good. Review of General Psychology 5(4):323-370

Blei D, Ng A, Jordan M (2003) Latent Dirichlet allocation. Journal of Machine Learning Research 3:9931-1022

Budak C, Agrawal D, El Abbadi A (2011) Limiting the spread of misinformation in social networks. In: Proceedings of the 20th International Conference on World Wide Web (WWW'11), pp 665-674

Cheng J, Adamic L, Dow PA, Kleinberg JM, Leskovec J (2014) Can cascades be predicted? In: Proceedings of the 23rd International Conference on World Wide Web (WWW'14), pp 925-936

Cheng J, Adamic LA, Kleinberg J, Leskovec J (2016) Do cascades recur? In: Proceedings of the 25th International Conference on World Wide Web (WWW'16), pp 671681

De Domenico M, Lima A, Mougel P, Musolesi M (2013) The anatomy of a scientific rumor. Scientific Reports 3:2980:1-2980:9

Dwass M (1960) Some $k$-sample rank-order tests. In: Contributions to Probability and Statistics, Stanford University Press, pp 198-202

Ferrara E, Yang Z (2015) Quantifying the effect of sentiment on information diffusion in social media. PeerJ Computer Science 1:e26

Gonçalves P, Araújo M, Benevenuto F, Cha M (2013) Comparing and combining sentiment analysis methods. In: Proceedings of the first ACM Conference on Online Social Networks (COSN'13), pp 27-38

Gruzd A, Doiron S, Mai P (2011) Is happiness contagious online? A case of Twitter and the 2010 Winter Olympics. In: Proceedings of the 44th Hawaii International Conference on System Sciences (HICSS'11), pp 1-9

Hansen L, Arvidsson A, Nielsen F, Colleoni E, Etter M (2011) Good friends, bad news - Affect and virality in Twitter. Future Information Technology 185:34-43

Hong L, Convertino G, Chi EH (2011a) Language matters in Twitter: A large scale study. In: Proceedings of the 5th International AAAI Conference on Weblogs and Social Media (ICWSM'11), pp 518-521

Hong L, Dan O, Davison B (2011b) Predicting popular messages in Twitter. In: Proceedings of the 20th International Conference on World Wide Web (WWW'11), pp 57-58

Karsai M, Kaski K, Barabási AL, Kertész J (2012) Universal features of correlated bursty behaviour. Scientific Reports 2:397:1-397:7

Kempe D, Kleinberg JM, Tardos E (2003) Maximizing the spread of influence through a social network. In: Proceedings of the 9th ACM SIGKDD International Conference on Knowledge Discovery and Data Mining (KDD’03), pp 137-146

Kontopoulos E, Berberidis C, Dergiades T, Bassiliades N (2013) Ontology-based sentiment analysis of Twitter posts. Expert Systems with Applications 40(10):40654074

Kupavskii A, Ostroumova L, Umnov A, Usachev S, Serdyukov P, Gusev G, Kustarev A (2012) Prediction of retweet cascade size over time. In: Proceedings of the 21st ACM International Conference on Information and Knowledge Management (CIKM'12), pp 2335-2338

Kwak H, Lee C, Park H, Moon S (2010) What is Twitter, a social network or a news media? In: Proceedings of the 19th International Conference on World Wide Web 
(WWW'10), pp 591-600

Liu B, Cong G, Xu D, Zeng Y (2012) Time constrained influence maximization in social networks. In: Proceedings of the IEEE 12th International Conference on Data Mining (ICDM'12), pp 439-448

Naveed N, Gottron T, Kunegis J, Alhadi A (2011) Bad news travel fast: A content-based analysis of interestingness on Twitter. In: Proceedings of the ACM Web Science Conference 2011 (WebSci'11), pp 1-7

Rosenthal S, Nakov P, Kiritchenko S, Mohammad SM, Ritter A, Stoyanov V (2015) SemEval-2015 task 10: Sentiment analysis in Twitter. In: Proceedings of the 9th International Workshop on Semantic Evaluation (SemEval'15), pp 451-463

Rozin P, Royzman EB (2001) Negativity bias, negativity dominance, and contagion. Personality and Social Psychology Review 5(4):296-320

Steel RGD (1960) A rank sum test for comparing all pairs of treatments. Technometrics 2(2):197-207

Stieglitz S, Dang-Xuan L (2012) Political communication and influence through microblogging — an empirical analysis of sentiment in Twitter messages and retweet behavior. In: Proceedings of the 45th Hawaii International Conference on System Science (HICSS'12), pp 3500-3509

Stieglitz S, Dang-Xuan L (2013) Emotions and information diffusion in social media-sentiment of microblogs and sharing behavior. Journal of Management Information Systems 29(4):217-247

Suh B, Hong L, Pirolli P, Chi E (2010) Want to be retweeted? Large scale analytics on factors impacting retweet in Twitter network. In: Proceedings of the 2nd IEEE International Conference on Social Computing (SocialCom'10), pp 177-184

Takamura H, Inui T, Okumura M (2005) Extracting semantic orientations of words using spin model. In: Proceedings of the 43rd Annual Meeting on Association for Computational Linguistics (ACL'05), pp 133-140

Takamura H, Inui T, Okumura M (2006) Extracting semantic orientations using spin model. IPSJ Journal 47(2):627637, (in Japanese)

Taylor SE (1991) Asymmetrical effects of positive and negative events: The mobilization-minimization hypothesis. Psychological Bulletin 110(1):67-85

Tsugawa S, Ohsaki H (2015) Negative messages spread rapidly and widely on social media. In: Proceedings of the 2015 ACM Conference on Online Social Networks (COSN'15), pp 151-160

Wen S, Jiang J, Xiang Y, Yu S, Zhou W, Jia W (2014) To shut them up or to clarify: Restraining the spread of rumors in online social networks. IEEE Transactions on Parallel \& Distributed Systems 25(12):3306-3316

Yang S, Kolcz A, Schlaikjer A, Gupta P (2014) Large-scale high-precision topic modeling on Twitter. In: Proceedings of the 20th ACM SIGKDD Conference on Knowledge Discovery and Data Mining (KDD'14), pp 1907-1916

Zhao K, Stehlé J, Bianconi G, Barrat A (2011) Social network dynamics of face-to-face interactions. Physical Review E 83(5):056,109 